\title{
Interference coordination of heterogeneous LTE systems using remote radio heads
}

\author{
Jaewon Kim, Donghyun Lee and Wonjin Sung*
}

\begin{abstract}
In this paper, we present an operational strategy to mitigate co-channel interference (CCI) by using geographically distributed remote radio heads (RRHs). The inter-node $\mathrm{CCl}$ becomes a dominant performance degradation factor for heterogeneous network (HetNet) systems. Recently, there are emerging attempts in Third Generation Partnership Project to adopt advanced techniques to Long Term Evolution Advanced systems to mitigate CCI problems for HetNet systems, namely, the coordinated multipoint transmission (CoMP). However, the CoMP scheme cannot control the $\mathrm{CCl}$ generated from outside coordination boundaries. To resolve this problem, we propose a partial activation strategy by using RRHs deployed near cell edge which results in moving coverage boundary effects. Based on Monte Carlo system level simulations, performance of the conventional strategies and the presented strategy is evaluated. Simulation results show that the proposed scheme outperforms the enhanced inter-cell interference coordination and CoMP schemes especially for users located near cell edge areas.
\end{abstract}

Keywords: Heterogeneous network, Remote radio head, Coordinated multipoint transmission, Enhanced inter-cell interference coordination, LTE-Advanced

\section{Introduction}

Limited capacity and coverage holes have been considered as major problems of conventional mobile cellular wireless systems which only consist of homogeneous macro base stations (BSs) [1]. To solve the problems, the conventional systems have been evolved to heterogeneous network (HetNet) systems for which different types of transmission nodes are newly deployed within a coverage area of the macro BS. The limited capacity can be efficiently increased by letting wireless resources of the macro BS be spatially reused in newly created coverage areas of the transmission nodes [2]. Moreover, by installing the nodes at the coverage holes of macro BSs, an outage probability of user equipments (UEs) decreases significantly [3].

Different types of transmission nodes have been implemented in current systems in forms of pico or femto BSs, relays, and remote radio heads (RRHs) [4]. In general, pico BSs are deployed by mobile service providers

*Correspondence: wsung@sogang.ac.kr

Department of Electronic Engineering, Sogang University, Seoul, 121-742, Korea with smaller coverage areas and transmission power compared to macro BSs. On the other hand, femto BSs are installed by users in their private indoor places, and their corresponding coverage areas are much smaller than those of pico and macro BSs. While pico and femto BSs create their own coverage areas, relay nodes are commonly used to extend the coverage area of macro BSs. RRHs are geographically distributed RF units of a transmission node which are connected to their own control units via optical fiber backhaul with nearly ideal conditions.

Performance gains of HetNet systems mainly come from two aspects, i.e., decreasing the outage probability and increasing the system capacity. However, newly installed HetNet transmission nodes may also increase the amount of co-channel interference (CCI) [5]. In the Long Term Evolution Advanced (LTE-Advanced) system, the inter-cell interference coordination (ICIC) and the enhanced ICIC (eICIC) schemes are adopted to mitigate CCI between transmission nodes [6]. The schemes prohibit undesirable scenarios such that adjacent nodes allocate the same time and frequency resources with full radiation power to UEs located near coverage boundaries.

\section{是 Springer}

(c) 2013 Kim et al: licensee Springer. This is an Open Access article distributed under the terms of the Creative Commons Attribution License (http://creativecommons.org/licenses/by/2.0), which permits unrestricted use, distribution, and reproduction in any medium, provided the original work is properly cited. 
Thus, these schemes utilize static or semi-static coordination among transmission nodes through X2 interface backhaul for the management of time, frequency, and power resources.

The coordinated multipoint transmission (CoMP) is a promising technique for inter-cell CCI mitigation, which has been included in Third Generation Partnership Project (3GPP) Release 11 specifications [5,6]. Currently, two types of CoMP schemes are considered which are known as the coordinated scheduling/coordinated beamforming scheme and the joint processing (JP) scheme. For JP, multiple transmission nodes act as a single transmitter with geographically distributed antennas for which scheduling information, transmission data, and the channel state information (CSI) are fully exchanged through the backhaul network. Compared to eICIC, CoMP is a far more direct and dynamic solution to reduce CCI.

However, conventional methods cannot be a complete solution for interference problem in HetNets. Since some part of radio resources or the transmission power should be reserved for interference mitigation, there exists inevitable performance loss in spectral efficiency in the cases of ICIC and eICIC. For CoMP, performance is largely dependent on capabilities of the backhaul network, which can be a serious bottleneck in obtaining the desired gain [7]. Moreover, CCI problems still exist for the UEs located at coverage boundaries of a cluster of transmission nodes participating in the CoMP $[8,9]$.

In this paper, we propose an interference mitigation strategy using geographically distributed RRHs in HetNet systems. Within a cell, a macro BS and RRHs share the same cell identification (CID) and participate in CoMP to mitigate intra-cell interference. We introduce virtual cell coverage which is the union of two time-varying coverage areas. By means of designing the virtual coverage area to be larger than the area where scheduling candidate UEs are located, our proposal also efficiently mitigates inter-cell interference from the transmission nodes with different CIDs. Performance of the conventional and presented strategies is evaluated by Monte Carlo system level simulations to demonstrate the effectiveness of the proposal.

This paper is organized as follows. In Section 2, the system model is presented which consists of 57 cells and 19 cell groups. We consider HetNet systems where the macro BSs and the RRHs are referred to as different types of transmission nodes. Section 3 presents conventional interference management methods including eICIC and CoMP. The proposed interference management method in HetNet is described in Section 4. Performance of the presented strategy is evaluated and analyzed in Section 5 based on the numerical results using Monte Carlo simulations. Concluding remarks are given in Section 6.
For notational simplicity, we let $\mathbf{X}=\left[\mathbf{x}_{i, j}\right]_{i \in A, j \in B}$ denote the matrix generated by column-wise stacking for all $i$ in $A$ and row-wise stacking for all $j$ in $B$. Expressions $\left[\mathbf{x}_{i, j}\right]_{i \in A}$ and $\left[\mathbf{x}_{i, j}\right]_{j \in B}$ respectively denote column-wise stacking and row-wise stacking.

\section{System model}

We consider the cellular system shown in Figure 1 which includes a total of 57 cells, where each cell is the hexagonal sector coverage area as defined in $[10]^{\mathrm{a}}$ and is covered by the macro BS with multiple antenna elements having vertical and horizontal beam patterns. The dashed arrow indicates the center of the horizontal beam pattern for each macro BS. A cell group is the set of three adjacent cells surrounded by red lines in Figure 1. Macro BSs are co-located at the center of each cell group.

For homogeneous network systems, macro BSs are the only type of transmission node. On the other hand, RRHs are another type of transmission nodes for HetNet systems. The two types of transmission nodes are differentiated by various physical parameters including transmit power, antenna height, and antenna beam patterns. The backhaul network within a cell between the RRHs and the macro BS is connected via optical fibers which are assumed to exhibit nearly ideal conditions of zero delay and infinite bandwidth. Hence, in terms of the capability to share information through the backhaul network, geographically distributed transmission nodes can participate in coordination of transmissions and scheduling with no penalty in performance.

Let $M_{i}$ indicate the number of antennas and $P_{i}$ indicate the maximum transmit power of the $i$ th transmission

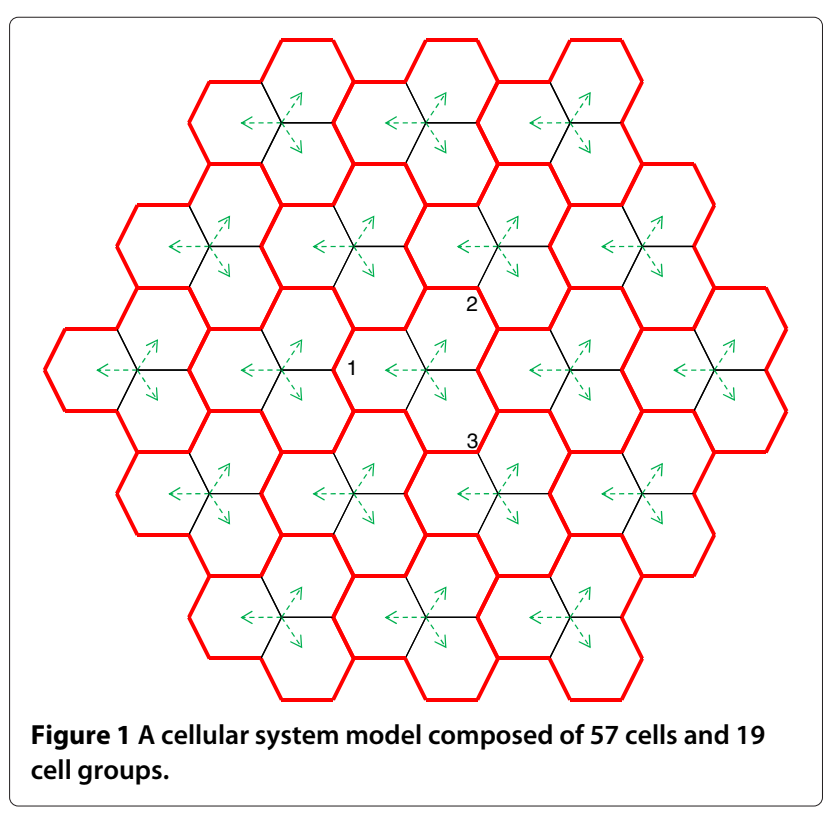


node. Let us consider the $k$ th UE with single antenna in the $n$th cell. Then, the received signal of the UE can be represented as

$$
y_{k}=\sum_{i \in A_{n}} \sqrt{P_{i} s_{k, i}} \mathbf{h}_{k, i}^{H} \mathbf{x}_{i}+\sum_{j \neq n} \sum_{i \in A_{j}} \sqrt{P_{i} s_{k, i}} \mathbf{h}_{k, i}^{H} \mathbf{x}_{i}+z_{k}
$$

where $\mathbf{h}_{k, i}$ and $\mathbf{x}_{i}$ are $M_{i} \times 1$ complex vectors which respectively represent the short-term CSI vector and the transmit signal vector from the $i$ th node. The elements in the short-term CSI vector follow the independent and identically distributed (i.i.d.) complex normal distributions with zero mean and unit variance. The additive white Gaussian noise is denoted by $z_{k}$ whose elements also follow the i.i.d. complex normal distributions with zero mean and $\sigma_{0}^{2}$ variance.

The long-term CSI of the link from the $i$ th node to the $k$ th UE is denoted by $s_{k, i}$ which includes the effects of path loss, shadowing, and antenna beam patterns. For simulations in Section 5, the urban macro and the urban micro models are respectively applied to the channels of the macro BSs and the RRHs, as specified in [11]. Both the horizontal and vertical antenna beam patterns are applied to the links of macro BS, and the vertical antenna beam pattern is used for the RRHs with omni-directional antennas. Parameters for antenna beam patterns are per 3GPP Case 1 (Table A.2.1.1-2 in [12]). Specific parameters regarding the system and signal models are summarized in Table 1.

\section{Table 1 Simulation parameters}

\begin{tabular}{ll}
\hline Description & Parameters \\
\hline Transmission node height & $25 \mathrm{~m}$ for macro BSs \\
& $10 \mathrm{~m}$ for RRHs \\
& $46 \mathrm{dBm}$ for macro BSs \\
Transmission power & $30 \mathrm{dBm}$ for RRHs \\
& Horizontal and vertical patterns for macro BSs \\
Beam patterns & Vertical pattern only for RRHs \\
& Single receive antenna for UEs \\
Number of antennas & Eight transmit antennas for macro BSs \\
& Two transmit antennas for RRHs \\
& 57 cells and 19 cell groups \\
Inter macro BS distance D $=500 \mathrm{~m}$ \\
Node placement & Uniformly distributed $K=30$ UEs per cell \\
& $N=4$ (default) \\
Number of RRHs per cell & $N=1,2,3$ (optional) \\
Number of simulations & $N_{\text {sim }}=100$ \\
& $N$ time $=3,000$ ( $\alpha=1,000)$ \\
Traffic model & Full buffer \\
\hline &
\end{tabular}

A scheduling region is defined as the area where scheduling candidate UEs are located. A group of transmission nodes sharing the same CID within a cell has its own scheduling region, and the corresponding scheduling is made independently to the other regions. Among the UEs within a scheduling region, up to $L=8$ UEs can be selected for the multiuser multiple-input multiple-output (MU-MIMO).

\section{Conventional interference management methods (in HetNet)}

\section{1 elCIC}

Typically, a UE chooses its serving node to be the one with the strongest received power. Due to the large difference in transmit power of a macro BS and RRHs, the coverage of RRHs is much smaller than that of a macro BS [13]. In this regard, a significantly smaller number of UEs select the RRHs as their own serving nodes, which yields an imbalance in traffic load between the two types of transmission nodes. For traffic load balancing, LTE-Advanced systems adopt cell range expansion (CRE) to low-power transmission nodes by increasing preference values in the process of serving node selection.

Upon applying CRE, however, some UEs may choose RRHs as their serving nodes even though channel gains of RRHs are smaller than channel gains of macro BSs. These UEs suffer from strong interference from macro BSs, and their channel conditions are degraded. The eICIC reduces the interference from macro BSs to the UEs served by RRHs $[4,14]$. Through the power control of the macro BSs, their transmission is suspended in the designated time duration. In LTE-Advanced specifications, this duration is called the almost blank subframe (ABS).

In performance evaluations of eICIC, we assume that the macro BSs and the RRHs are independent transmission nodes with different CIDs. Therefore, each transmission node has its own scheduling region which is identical to the coverage area of the transmission node. To mitigate the inter-node interference, transmission of the macro BS is periodically muted by using the $\mathrm{ABS}$ in a synchronous manner. The time duration of the ABS over the entire subframes is controlled by the activation ratio $\tau$. During the ABS, the transmission of control, data, and reference signal from the macro BS is completely eliminated.

For $\tau$ portion of entire subframes, the transmission signal of the $i$ th node is given by

$$
\mathbf{x}_{i}=\sum_{k \in S_{i}} \mathbf{w}_{i, k} \sqrt{q_{k}} d_{k}
$$

for all $i$ 's within a cell where $\mathbf{w}_{i, k}$ denotes a beamforming vector for the $k$ th UE and $S_{i}$ is an index set of scheduled UEs for the $i$ th transmission node. A data symbol 
for the $k$ th UE is represented by $d_{k}$, and its power control scalar is written as $q_{k}$. Defining the concatenated channel matrix from the $i$ th node to the scheduled UEs as $\mathbf{H}_{i}=\left[\sqrt{P_{i} s_{k, i}} H \text { k,i }\right]_{k \in S_{i}}$, the corresponding zero-forcing beamforming matrix $\mathbf{W}_{n, i}=\left[\mathbf{w}_{i, k}\right]_{k \in S_{n, i}}$ is written as

$$
\mathbf{W}_{n, i}=\mathbf{H}_{n, i}^{H}\left(\mathbf{H}_{n, i} \mathbf{H}_{n, i}^{H}\right)^{-1}
$$

which cancels out the CCI generated only within the coverage area of the $i$ th transmission node. To limit the transmit power of the $i$ th node under $P_{i}$, which is called the per-node power constraint, the power allocation variable is set to

$$
q_{k}=\left|S_{i}\right|^{-1}\left\|\mathbf{w}_{i, k}\right\|^{-2}
$$

which guarantees $E\left\|\mathbf{x}_{i}\right\|^{2}=1$. For the ABS, the transmission signal is given by $x_{i}=0$ for the index $i$ assigned to the macro BS, while the transmission signals from the RRHs exhibit the same format as the one in Equation 2.

\subsection{CoMP}

For CoMP, we consider the case of all transmission nodes within a cell, one macro BS and $N$ RRHs, participating in joint transmission. Let us define $A_{n}$ as the index set of transmission nodes within the $n$th cell. By letting all the nodes in $A_{n}$ share the same CID, the RRHs act as geographically distributed antennas of the macro BS. The coverage area of the set is defined as a single scheduling region, and coordination in transmitting signals and scheduling UEs is constrained among the transmission nodes within a set. Thus, up to $L$ UEs can be scheduled for the MU-MIMO transmission over the entire cell.

Let $S_{n}$ be the set of scheduled UEs by the $n$th cell, which is also assumed to be given. Then, the transmit signals from the transmission nodes within the cell can be written as

$$
\left[\begin{array}{c}
\vdots \\
\mathbf{x}_{i} \\
\vdots
\end{array}\right]_{i \in A_{n}}=\left[\begin{array}{c}
\vdots \\
\sum_{k \in S_{n}} \mathbf{w}_{i, k} \sqrt{q_{k}} d_{k} \\
\vdots
\end{array}\right]_{i \in A_{n}}
$$

Let us define $\mathbf{W}_{n}=\left[\mathbf{w}_{i, k}\right]_{i \in A_{n}, k \in S_{n}}$ and $\mathbf{H}_{n}=$ $\left[\sqrt{P_{i} s_{k, i}} \mathbf{h}_{k, i}^{H}\right]_{k \in S_{n}, i \in A_{n}}$ respectively as the concatenated beamforming and channel matrices from the transmission nodes in $A_{n}$ to the scheduled UEs in $S_{n}$. To eliminate the CCI among the transmission nodes, the concatenated beamforming matrix is determined by

$$
\mathbf{W}_{n}=\mathbf{H}_{n}^{H}\left(\mathbf{H}_{n} \mathbf{H}_{n}^{H}\right)^{-1} .
$$

To satisfy the per-node power constraints, we let the power allocation variable be

$$
q_{k}=\frac{1}{\mu_{n} \sum_{i \in A_{n}}\left\|\mathbf{w}_{i, k}\right\|^{2}}
$$

where the normalization value is given by $\mu_{n}=$ $\max _{i \in A_{n}}\left(\sum_{k \in S_{n}} \frac{\left\|\mathbf{w}_{i, k}\right\|^{2}}{\sum_{j \in A_{n}}\left\|\mathbf{w}_{j, k}\right\|^{2}}\right)[15]$. This solution guarantees that at least one transmission node in $A_{n}$ can satisfy the per-node constraint with equality and nullifies the intra-cell CCI.

\section{Proposed interference management method (in HetNet)}

Unlike conventional eICIC, transmission from RRHs is periodically deactivated, whereas transmission from macro BSs is always being activated. For $\tau$ portion of the entire subframes, all transmission nodes in $A_{n}$ participate in CoMP joint transmission to mitigate the intra-cell CCI. In that time period, the transmission signal is generated as the form in Equation 5. For the remaining $1-\tau$ portion of subframes, the transmission from the RRHs is muted, and only the signal from the macro BS is transmitted as given by Equation 2.

Due to the periodic activation of RRHs, the coverage area for the set $A_{n}$ changes in time. When the RRHs are deactivated, the corresponding coverage area is shown in the left-hand side of Figure 2a which is identical to the case of homogeneous network systems. On the other hand, when the RRHs are activated, the corresponding coverage area changes as indicated in the right-hand side of Figure 2a. In this case, the coverage area is identical to the conventional CoMP case. Since we assume that the entire network is synchronized in time, the variations in the coverage area of the set $A_{n}$ are identical for all $n$.

We propose a concept of virtual coverage area of the set $A_{n}$ which is a union of the two coverage areas. Since the virtual coverage area is the union of two different shapes, it becomes larger than individual areas. For CoMP transmissions, all transmission nodes in $A_{n}$ share the same CID. To determine the scheduling region of the proposed scheme, a UE measures two signal-to-interference plus noise ratio (SINR) values, $\gamma_{1, n}$ and $\gamma_{2, n}$, for each $A_{n}$ which correspond to the cases wherein the RRHs are activated and deactivated, respectively. Based on the two values, the UE computes two spectral efficiency values, $\eta_{1, n}=$ $\tau \log _{2}\left(1+\gamma_{1, n}\right)$ and $\eta_{2, n}=(1-\tau) \log _{2}\left(1+\gamma_{2, n}\right)$. The UE selects the larger spectral efficiency value as the expected rate of set $A_{n}$. For all sets in the system, the serving set is chosen as the one with the largest expected rate. Then, 


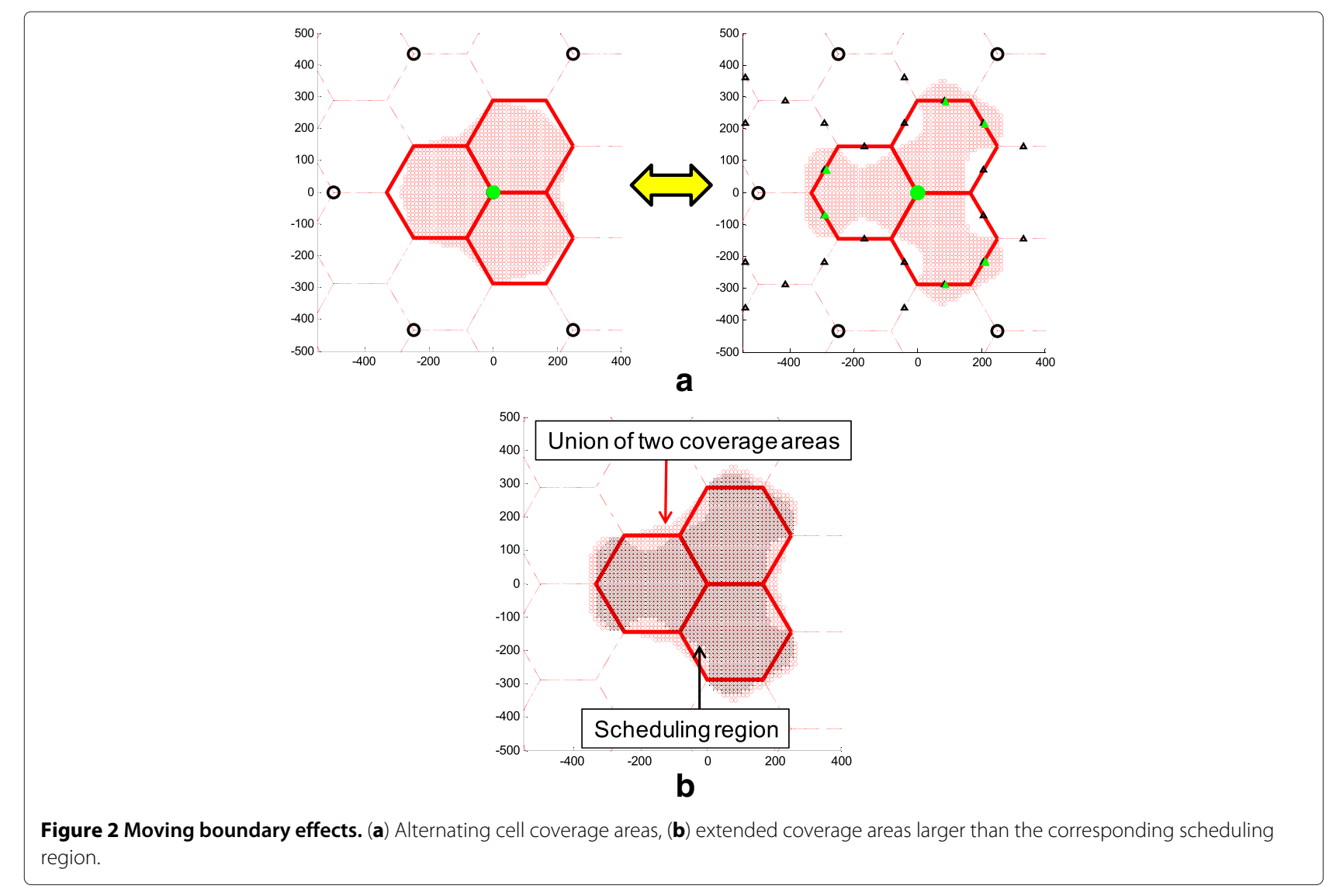

the UEs choosing set $A_{n}$ as their serving set become the scheduling candidate UEs for the set, and the corresponding scheduling region is the areas where the scheduling candidate UEs are located, as shown in Figure 2b.

The proposed scheme with dynamically activated RRHs results in moving boundaries of the cell coverage area. The moving boundary effect can help prevent the case wherein a certain UE is located at the edge of the cell during the whole transmission time. Therefore, the proposed scheme can mitigate inter-cell CCI by letting no scheduling candidate UE be at the boundary of the virtual cell coverage area.

\section{Performance evaluations}

The UEs scheduled by macro cells, numbered 1, 2, and 3 in Figure 1, are considered for performance evaluations. Along with the SINR, the UE throughput is adopted as a major performance metric [11]. The UE throughput value is also utilized to determine individual UE weight values for proportional fairness. Greedy sequential UE scheduling is independently conducted within the cooperation area under the weighted sum-rate maximization criterion [16].

Coverage probability is defined as the probability that a UE has a larger SINR value than the given threshold value.
In Figure 3, the coverage probability is presented with respect to the number of RRHs per cell. This figure shows that the overall coverage probability improved. In particular, the low-tier UE performance is significantly enhanced according to the increase of the number of RRHs. This shows that the SINR gain of the cell boundary can be

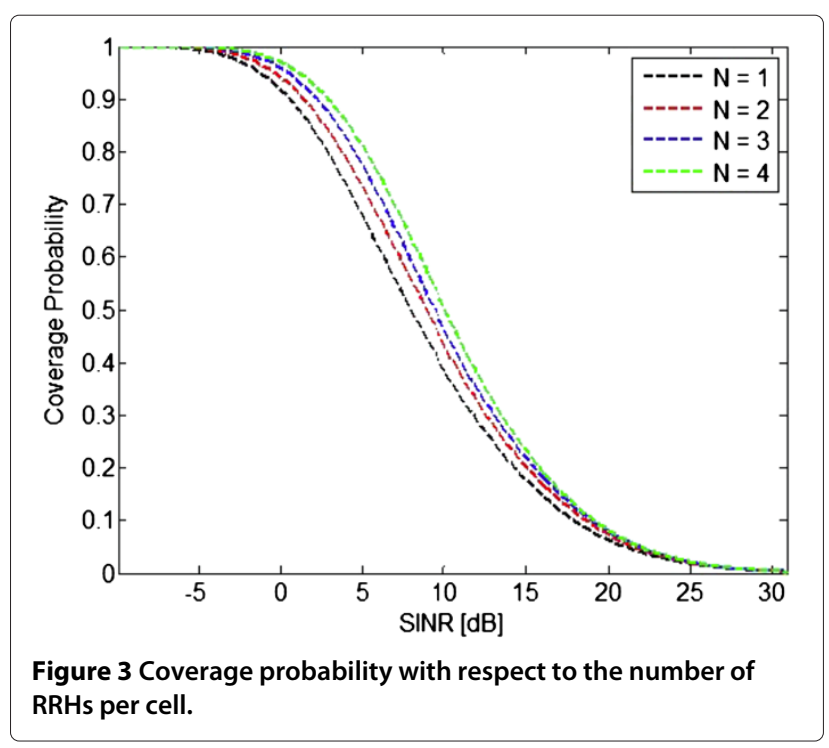




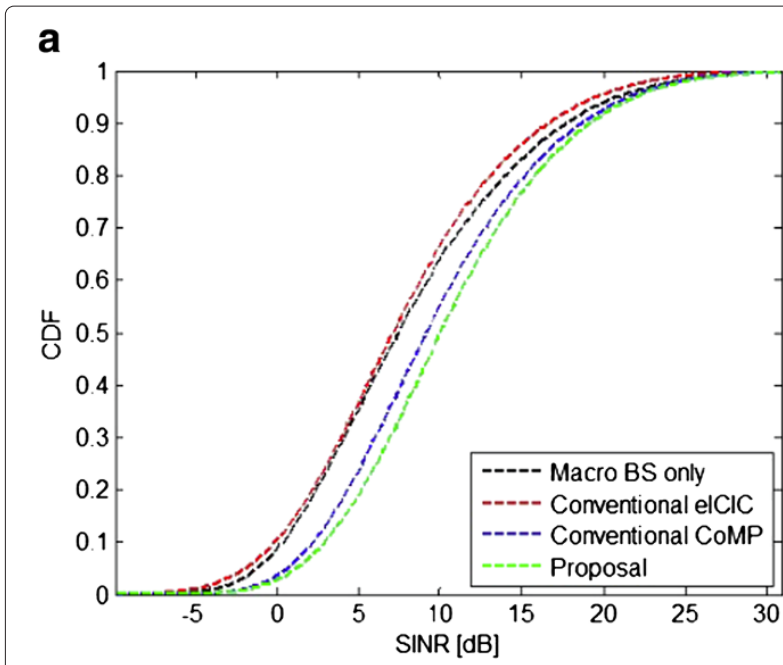

b

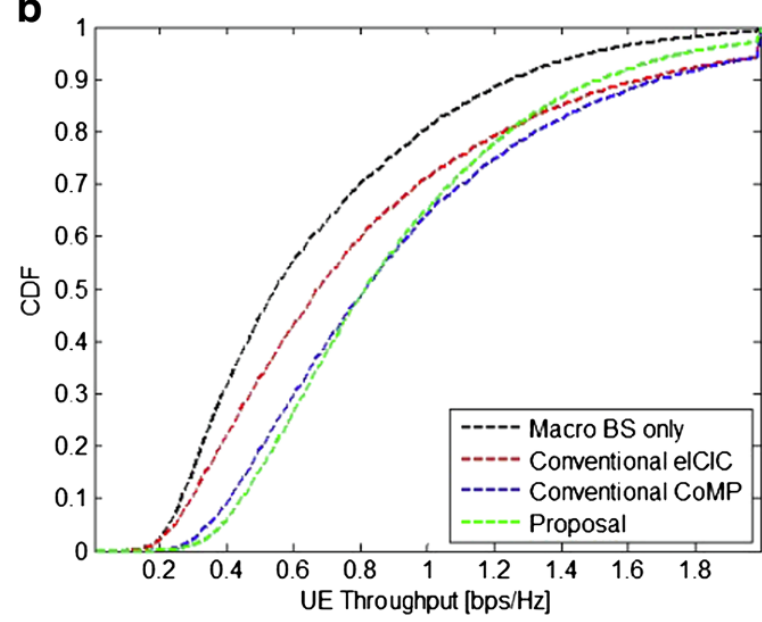

Figure 4 Comparison of cooperation strategies. Where $N=4$ RRHs are positioned on the cell boundary of each macro BS using activation ratio $\tau=0.7$. (a) Performance in SINR, (b) performance in UE throughput.

achieved by signal transmission from RRHs to UEs located in cell boundary areas.

In Figure 4, the performance of the proposed scheme is compared to the conventional schemes in terms of SINR and UE throughput. The conventional eICIC scheme shows better UE throughput performance (about 122.4\% on average) than the homogeneous system with the macro BS only. Through the comparison of conventional eICIC and CoMP, it is observed that the effect of intra-cell interference cancellation by the ZF produces performance gain. A difference in performance between the proposed scheme and the conventional CoMP scheme results from the decrease of inter-cell interference due to moving boundary effects. The proposed scheme demonstrates improved performance over conventional CoMP, including $110 \%$ UE throughput gain for low $5 \%$ UEs in terms of throughput.

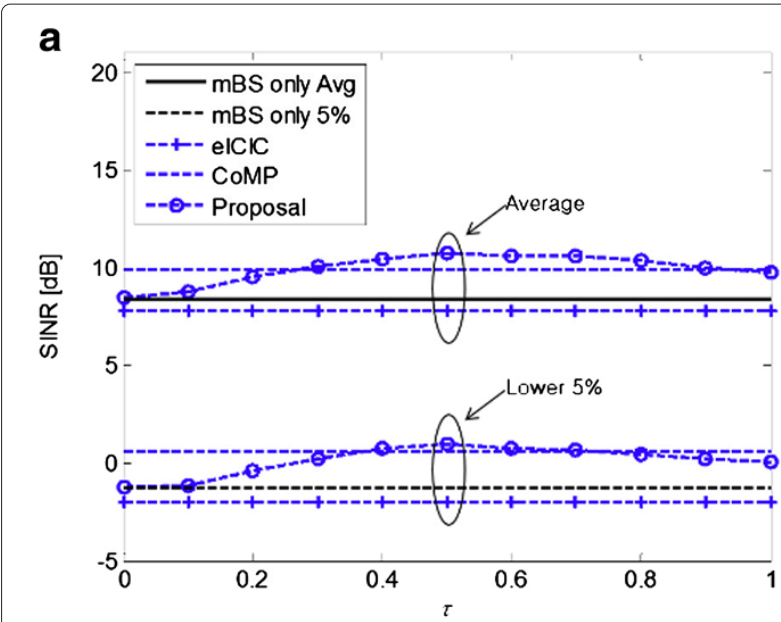

b

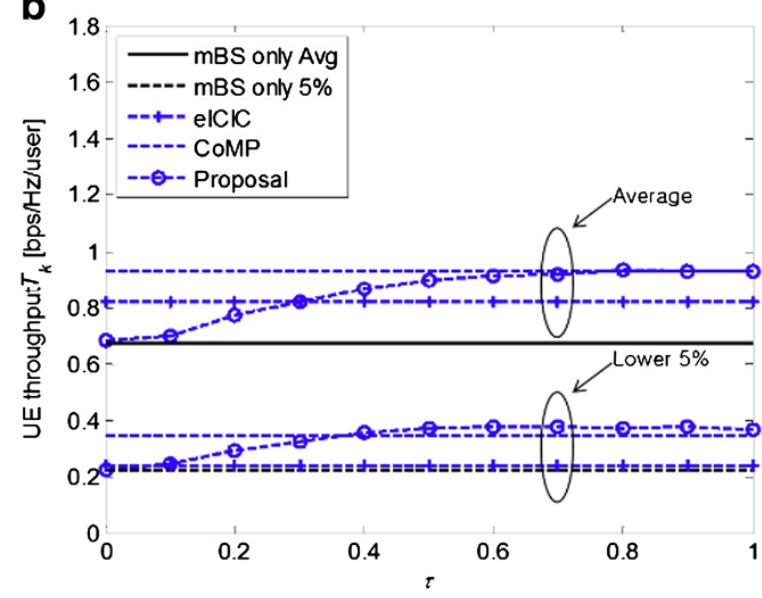

Figure 5 Comparison of operation strategies. Where $N=4 R R H s$ are positioned on a cell boundary of each macro BS. (a) Performance in SINR, (b) performance in UE throughput.

In Figure 5, the proposed scheme and the conventional schemes including the macro BS only, eICIC, and CoMP are compared with respect to activation ratio $\tau$. In the case of $\tau=0$, the proposed scheme shows the same performance with that of the macro BS-only scheme because all RRHs are deactivated. The proposed scheme, on the other hand, exhibits the same performance with that of the conventional CoMP scheme when $\tau=1$ because all RRHs are activated. Through the selection of a proper $\tau$ value, we can maximize both the SINR performance and UE throughput performance. When the lower 5\% UE throughput is chosen as the main performance metric, the optimum value of $\tau=0.7$ produces nearly $280 \%$ UE throughput gain over the homogeneous system.

Figure 6 presents different scenarios of RRH deployment. The positions of RRHs are marked by circles which represent different cell group affiliations. In Figure 6a, RRHs are randomly located in arbitrary positions within a 


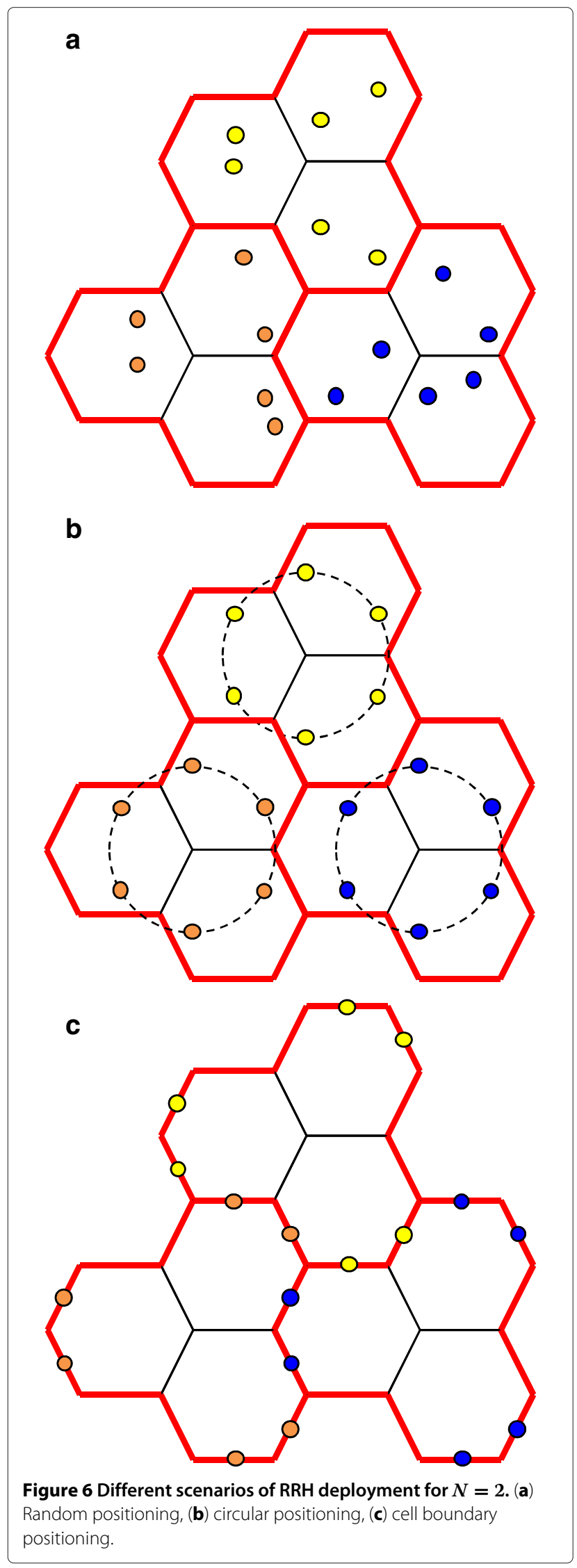

cell according to a uniform distribution. Since UEs are also randomly located according to a uniform distribution, the average distance between a UE and a transmission node becomes smaller as $N$ increases. For $N_{C}=3 N$, RRHs on the same circle, their positions are uniformly determined as shown in Figure 6b where the distance of two adjacent RRHs is set to $d_{1}$. Distance $d_{1}$ monotonically increases with respect to the radius $R$ of the circles. On the other hand, let us define $d_{2}$ as the minimum distance between RRHs located on different circles. Since the inter-macro BS distance is fixed, distance $d_{2}$ monotonically decreases with respect to the radius $R$. Therefore, the optimal value of $R$ can be obtained by letting $d_{1}=d_{2}$. We can also consider RRHs located at the boundary of cell groups, as shown in Figure 6c, where the amount of inter-cell CCI is maximum. Since adjacent cell groups share common cell boundaries, positions of RRHs are divided into three different cell groups to prevent duplicated RRH positions.
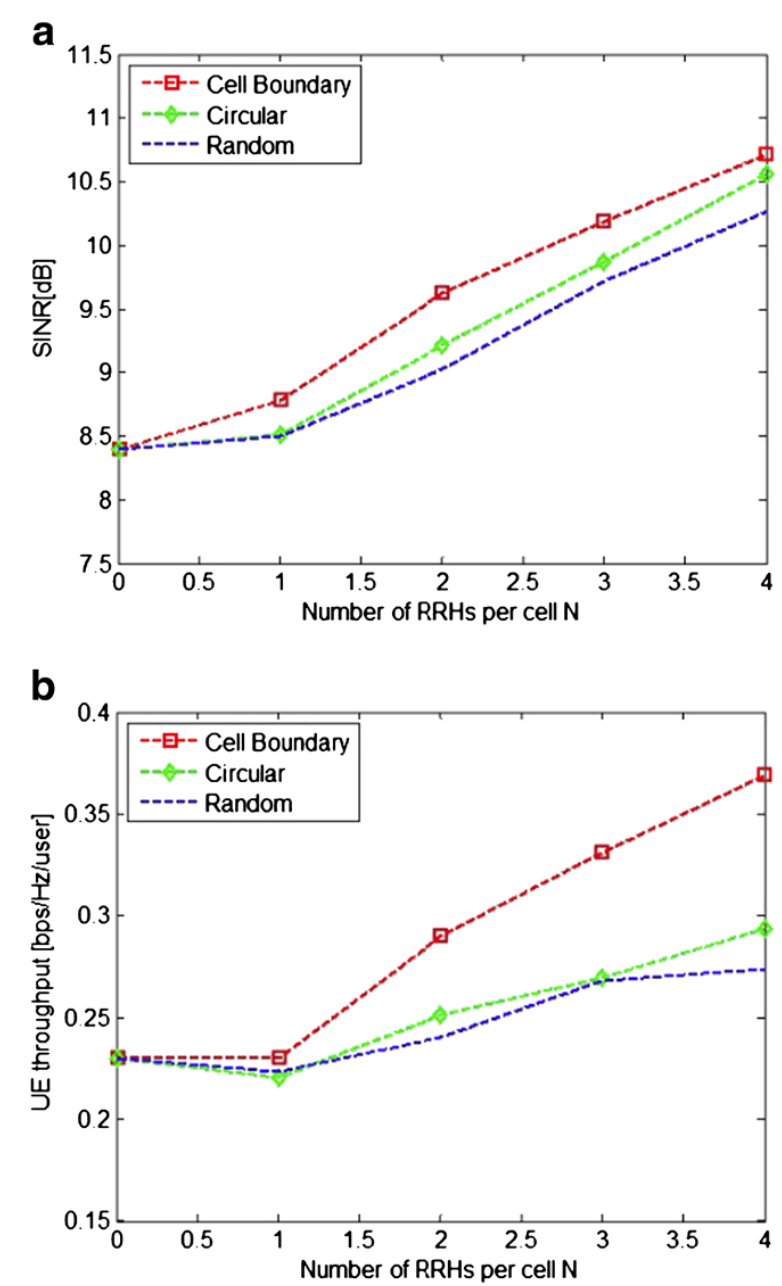

Figure 7 Comparison of RRH deployment scenarios. (a)

Performance in average SINR, (b) performance in lower 5\% UE throughput. 
For a given cell group boundary, $N_{C}$ RRHs which are affiliated to three different adjacent cell groups are deployed. As in the circular positioning scheme, potential locations of RRHs are determined to maximize the minimum distance between two adjacent RRHs.

In Figure 7, the average SINR and the lower 5\% UE throughput performance of different positioning scenarios are presented for the increasing value of $N$. The proposed scheme expands the virtual cell coverage through the moving cell boundary effect utilizing partial activation of RRHs in a time division fashion. In this way, we can ensure that the scenario wherein RRHs are located at the cell boundary provides enhanced performance over other scenarios.

\section{Conclusions}

In this paper, we presented an interference mitigation strategy to mitigate the intra-cell and inter-cell CCI problems via utilization of RRHs combined with CoMP. Motivated by the conventional eICIC and CoMP schemes, the proposed scheme not only utilizes CoMP transmission to eliminate intra-cell CCI but also partially activates celledge-located RRHs to control inter-cell CCI. In particular, we proposed the concept of moving boundary and virtual cell coverage, which can be efficiently adopted in the current specification of LTE-Advanced systems. Simulation results confirm the performance improvement of the proposal, and the reduction of inter-cell CCI gives a significant performance gain especially for the low-tier UEs near cell boundary areas.

\section{Endnote}

${ }^{a}$ Unlike 3GPP where cell is defined as a coverage area of any transmission node, we only use the term 'cell' as a hexagonal area of macro BS. Therefore, interference between the macro BS and the RRHs refers to intra-cell interference or inter-node interference.

\section{Competing interests}

The authors declare that they have no competing interests.

\section{Acknowledgements}

This work was supported by a grant from the National Research Foundation of Korea (NRF) funded by the Korean government (MEST) (no. 2011-0016146)

Received: 3 June 2012 Accepted: 3 April 2013

Published: 26 April 2013

\section{References}

1. M Rahman, H Yanikomeroglu, Enhancing cell-edge performance: a downlink dynamic interference avoidance scheme with inter-cell coordination. IEEE Trans. Wireless Commun. 9(4), 1414-1425 (2010)

2. D Astely, E Dahlman, A Furuskar, Y Jading, M Lindstrom, S Parkvall, LTE: the evolution of mobile broadband. IEEE Commun. Mag. 47(4), 44-51 (2009)

3. A Damnjanovic, J Montojo, Y Wei, T Ji, T Luo, M Vajapeyam, T Yoo, O Song, D Malladi, A survey on 3GPP heterogeneous networks. IEEE Wireless Commun. Mag. 18(3), 10-21 (2011)

4. D Lopez-Perez, I Guvenc, G de la Roche, M Kountouris, T Quek, J Zhang, Enhanced intercell interference coordination challenges in heterogeneous networks. IEEE Wireless Commun. Mag. 18(3), 22-30 (2011)

5. M Sawahashi, Y Kishiyama, A Morimoto, D Nishikawa, M Tanno, Coordinated multipoint transmission/reception techniques for LTE-advanced [Coordinated and distributed MIMO]. IEEE Wireless Commun. Mag. 17(3), 26-34 (2010)

6. R Irmer, H Droste, P Marsch, M Grieger, G Fettweis, S Brueck, HP Mayer, L Thiele, $V$ Jungnickel, Coordinated multipoint: concepts, performance, and field trial results. IEEE Commun. Mag. 49(2), 102-111 (2011)

7. 3rd Generation Partnership Project, TSG RAN: (E-UTRA). 3GPP TR 36.819 version 11.1.0 Coordinated multi-point operation for LTE physical layer aspects (Release 11) (2011). http://www.3gpp.org/ftp/Specs/html-info/ 36819.htm

8. P Marsch, G Fettweis, in IEEE International Conference on Communications (ICC). Static clustering for cooperative multi-point (COMP) in mobile communications (Kyoto, 5-9 June 2011)

9. J Zhang, R Chen, J Andrews, A Ghosh, R Heath, Networked MIMO with clustered linear precoding. IEEE Trans. Wireless Commun. 8(4), 1910-1921 (2009)

10. P Marsch, G Fettweis, Coordinated Multi-Point in Mobile Communications From Theory to Practice. (Cambridge University Press, Cambridge, 2011)

11. IT U, Guidelines for evaluation of radio technologies for IMT-Advanced. TS ITU-R, M.2135 (2008). http://itu.int/publ/R-REP-M.2135-2008/en

12. PartnershipProject 3rd Generation, TSG RAN: (E-UTRA): 3GPP TR 36.814 version 9.0.0 Evolved universal terrestrial radio access (E-UTRA). Further advancements for E-UTRA physical layer aspects (Release 11) (2010)

13. $\mathrm{M} \mathrm{Li}$, J Zhou, L Liu, X She, L Chen, in IEEE International Conference on Communications Workshops (ICC Workshops). Secondary serving cell selection for heterogeneous network with RRH deployment (Kyoto, 5-9 June 2011)

14. Y Hong, N Lee, B Clerckx, in IEEE GLOBECOM Workshops (GC Wkshps). System level performance evaluation of inter-cell interference coordination schemes for heterogeneous networks in LTE-A system (Miami, 6)

15. H Zhang, H Dai, Cochannel interference mitigation and cooperative processing in downlink multicell multiuser MIMO networks. EURASIP J. Wirel. Commun. Netw. 2004(2), 222-235 (2004)

16. C Guthy, W Utschick, R Hunger, M Joham, Efficient weighted sum rate maximization with linear precoding. IEEE Trans. Signal Process. 58(4) (2010)

doi:10.1186/1687-6180-2013-90

Cite this article as: Kim et al:: Interference coordination of heterogeneous LTE systems using remote radio heads. EURASIP Journal on Advances in Signal Processing 2013 2013:90.

\section{Submit your manuscript to a SpringerOpen ${ }^{\mathcal{O}}$ journal and benefit from: \\ - Convenient online submission \\ - Rigorous peer review \\ - Immediate publication on acceptance \\ - Open access: articles freely available online \\ - High visibility within the field \\ - Retaining the copyright to your article}

Submit your next manuscript at $>$ springeropen.com 